\title{
Pro-resolving lipids offer a helping hand to antibiotics
}

Controlling the extent of the inflammatory response to infection is a balancing act: too little might facilitate infection, whereas too much can cause tissue damage. Reporting in Nature, Chiang et al. investigate the regulation (during infection) of specific fatty-acid-derived specialized pro-resolving mediators (SPMs), and demonstrate that they can lower the antibiotic doses needed to clear infection.

Using a model of murine peritonitis, the researchers first showed that Escherichia coli inoculation at $10^{5}$ colony-forming units (CFUs) evoked a self-limiting host response; polymorphonuclear neutrophil (PMN) infiltration peaked after 12 hours, with macrophage numbers increasing between 2 and 72 hours, and the bacteria were cleared by 24 hours. By contrast, mice that were challenged with higher titres ( $\left.10^{7} \mathrm{CFUs}\right)$ exhibited sustained PMN infiltration, limited macrophage numbers and sustained elevated bacterial counts.

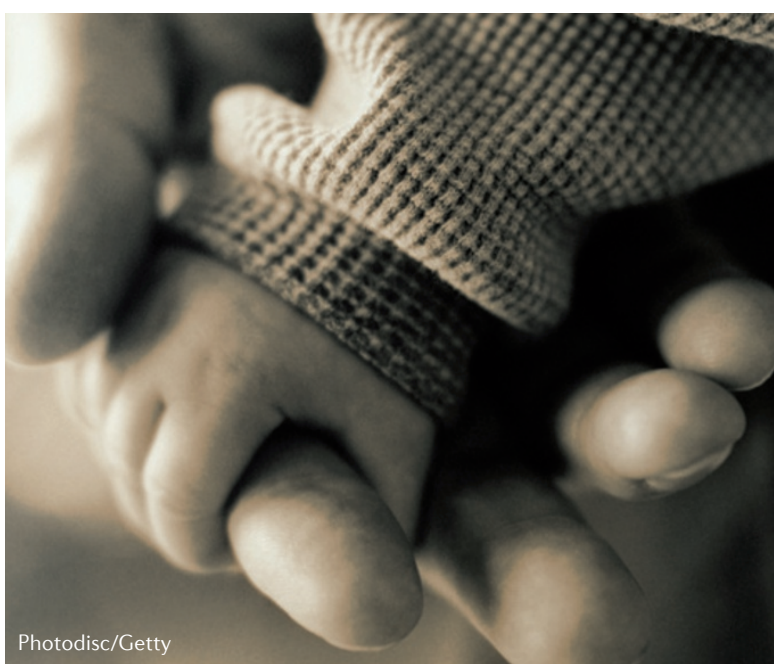

During the resolution of infection, SPMs such as D- and E-series resolvins and protectins are biosynthesized from essential fatty acids and actively participate in the resolution process. So, Chiang et al. then performed lipid mediator metabololipidomics on E. coli exudates from mice that were challenged with both titres to profile the SPMs. Although resolvin D5 and protectin D1 accumulated after 12 hours in self-resolving exudates, pro-inflammatory mediators such as leukotriene B4 predominated in exudates from high-titre $E$. coli infections. Germ-free mice also showed higher endogenous levels of protectin D1 and resolvin D1 than conventional mice.

What effect, then, do these SPMs have on E. coli infections? Administering physiological levels of resolvin D1 or D5 reduced blood and exudate bacterial counts in E. coli-challenged mice, concomitant with increasing phagocyte ingestion, and also reduced the levels of certain pro-inflammatory cytokines, thus preventing hypothermia and increasing survival. In human macrophages, resolvin D1, resolvin D5 and protectin D1 each promoted phagocytosis of E. coli. Notably, resolvin D5 activated the resolvin D1 receptor GPR32 (G protein-coupled receptor 32) to enhance this activity. Furthermore, the presence of resolvin D5 counter-regulated the increased expression in macrophages of several inflammation-related genes, such as tumour necrosis factor and nuclear factor- $\kappa \mathrm{B}$, that would normally be induced by E. coli. Resolvin D1, resolvin D5 and protectin D1 similarly stimulated antimicrobial mechanisms in PMNs in response to E. coli, enhancing bacterial ingestion and the production of reactive oxygen species.

Given these actions, Chiang et al. surmised that SPMs might enhance antibiotic treatment. On their own, resolvin D1 and ciprofloxacin both shortened the resolution interval (the time taken to reduce the number of PMNs from their maximum to $50 \%$ ) in mice challenged with $10^{5} \mathrm{CFUs}$ of $E$. coli, but together they further decreased this interval and also accelerated the onset of resolution. The synergistic effects extended to enhancing the phagocytic activity of macrophages, reducing host bacterial titres and selectively regulating host lipid mediators and cytokines. Resolvin D1, resolvin D5 and protectin D1 (in combination) also enhanced the effects of ciprofloxacin in infections with $10^{7}$ CFUs of E. coli. Similarly, this SPM combination further enhanced the clearance by vancomycin of Staphylococcus aureus from a model of skin infection.

So, in response to infection by Gram-negative or Grampositive bacteria, SPMs - such as resolvin D1, resolvin D5 and protectin D1 - produced by the host can accelerate resolution to enhance survival. When used in conjunction with antibiotics, these SPMs lower the required dose, which offers a potential new strategy in the battle against antibiotic resistance.

Katrin Legg

ORIGINAL RESEARCH PAPER Chiang, N. et al. Infection regulates pro-resolving mediators that lower antibiotic requirements. Nature $\mathbf{4 8 4}$, 524-528 (2012) 